\title{
TeV Halos: A New Class of TeV Sources Powered by Pulsars
}

\section{Tim Linden ${ }^{a}$}

${ }^{a}$ Stockholm University and The Oskar Klein Centre for Cosmoparticle Physics, Alba Nova, 10691 Stockholm, Sweden

E-mail: linden@fysik.su.se

Observations by the HAWC, H.E.S.S. and LHAASO telescopes have detected bright, spatially extended, and hard-spectrum $\gamma$-ray emission surrounding populations of young and middle-aged pulsars. The intensity of these sources indicates that pulsars efficiently convert a significant fraction of their spin-down power into the production of relativistic electron and positron pairs. Moreover, the surface brightness of this emission indicates that the $\mathrm{e}^{+} \mathrm{e}^{-}$pairs remain trapped in a $\sim 20$ pc region surrounding the pulsar for a considerable time - an observation that indicates that the diffusion constant surrounding energetic sources is significantly suppressed. Excitingly, recent observations have indicated that these sources continue to produce intense emission to energies exceeding $100 \mathrm{TeV}$. We discuss the current state of this field, and the potential for $\mathrm{TeV}$ halos to become a new tool capable of studying the most extreme processes in our universe.

$37^{\text {th }}$ International Cosmic Ray Conference (ICRC 2021)

July 12th - 23rd, 2021

Online - Berlin, Germany 


\section{Observations of TeV Halos}

Observations by the HAWC telescope in 2017 revealed something unexpected -the middle-aged pulsars, Geminga and Monogem, both produced bright and spatially extended $\gamma$-ray emission $[1,2]$ (see earlier results based on Monogem data by [3]). These observations significantly extended previous observations by H.E.S.S. which had found extended $\mathrm{TeV} \gamma$-ray emission surrounding younger sources (with typical ages of $\sim 20 \mathrm{kyr}$ ) [4]. While the Geminga and Monogem observations were consistent with the natural evolution of the younger pulsar population - unlike younger systems, their $\mathrm{TeV}$ emission could not be interpreted in the context of a supernova remnant or pulsar wind nebula (PWN) model. This lead to the reinterpretation of previous and existing systems - as belong to a new class of $\gamma$-ray emitters called "TeV halos". Initial TeV halo observations quickly illuminated several key results:

- (1) The high luminosity of these middle-aged systems could only be powered by the spindown luminosity of the central pulsar. A simple comparison between the observed spin-down power (from the P/Pdot diagram) and the measured TeV luminosity indicated that a significant fraction $\sim 10 \%$ of the pulsar spindown power must be converted into the acceleration of relativistic $\mathrm{e}^{+} \mathrm{e}^{-}$pairs [5-7].

- (2) The $\gamma$-ray spectrum indicated that this process efficiently accelerated electrons to energies of at least $100 \mathrm{TeV}$ (and possibly much higher), placing strong constraints on the potential dynamics of the electron acceleration (or re-acceleration) mechanism in the pulsar magnetosphere or surrounding PWN [1].

- (3) The large region encompassed by TeV halos indicates that both the magnetic field and interstellar radiation field energy densities are not influenced by the central source. Neither the pulsar, PWN, nor supernova remnant contain sufficient energy to significantly affect the surrounding environment. This implies that the $\mathrm{e}^{+} \mathrm{e}^{-}$energy loss times, dominated by a combination of synchrotron and inverse-Compton emission, are similar to their galactic averaged values [5].

- (4) The profile of the $\gamma$-ray emission indicates that the $\mathrm{e}^{+} \mathrm{e}^{-}$move diffusively throughout the region between $\sim 1-20 \mathrm{pc}$ from the central source. Combining this result with the high efficiency of $\mathrm{e}^{+} \mathrm{e}^{-}$production (Result 1), and the standard interstellar radiation field energy density (Result 3), indicates that $\mathrm{e}^{+} \mathrm{e}^{-}$pairs must remain confined for long timescales in order to lose most of their energy before leaving the $\sim 20 \mathrm{pc}$ region defined by the halo. This then implies that the diffusion constant within the TeV halo is significantly suppressed compared to the Galactic average. Notably, if particles propagated away from the central source with a diffusion constant equivalent to that of the galactic averaged value, the $\mathrm{TeV}$ halo emission would be spread over a region exceed $1 \mathrm{kpc}$ [8], with a surface brightness far below the sensitivity of current instruments [2,5]

These four key observations are distinct from any previously described source class, validating the existence of $\mathrm{TeV}$ halos as a new type of $\gamma$-ray source, which is dominantly detected at $\mathrm{TeV}$ energies (though we note that $\mathrm{TeV}$ halos should also be detectable in both $\mathrm{GeV} \gamma$-rays and from radio synchrotron observations). 


\section{TeV Halo Definition}

While the $\mathrm{TeV}$ halo emission surrounding Geminga and Monogem (and a handful of other pulsars that with ages exceeding $100 \mathrm{kyr}$ that are firmly displaced from their natal supernovae remannts) clearly demands a new physical mechanism, the classification of $\gamma$-ray emission surrounding younger pulsars is less clear. A single supernova event is expected to produce bright $\gamma$-ray emission from: (1) the hadronic interactions of high-energy protons with gas contained within the supernova remnant, (2) the leptonic interactions of $\mathrm{e}^{+} \mathrm{e}^{-}$pairs accelerated at (and confined by) the termination shock of the pulsar wind nebula, and (3) the leptonic emission from $\mathrm{e}^{+} \mathrm{e}^{-}$pairs diffusing through the broader TeV halo. Because all of these sources begin from a coincident location and have similar spectral and temporal variations, models must be used to distinguish each component.

At present, two different self-consistent definitions of $\mathrm{TeV}$ halo exist in the literature. The first, originally described in Ref. [6] and later clarified in Ref. [7] defines a TeV halo as:

Emission from relativistic $e^{+} e^{-}$pairs that are (at least originally) powered by the pulsars spindown power, and which are propagating diffusively (rather than advectively) through a region with standard magnetic field and interstellar radiation field strengths.

The second definition originally presented in Ref. [9] defines a TeV halo as:

Emission from relativistic $e^{+} e^{-}$pairs that are powered by the pulsars spindown power, and which are propagating diffusively through a region where the energy density of particles from the $\mathrm{TeV}$ halo falls below the energy density of the interstellar medium.

These two definitions are used to divide observed systems into a binary classification as either PWN or TeV halos. The second definition is stricter - as many systems with ages between 20 $100 \mathrm{kyr}$ exhibit diffusive $\mathrm{e}^{+} \mathrm{e}^{-}$propagation through the interstellar medium, but maintain $\mathrm{e}^{+} \mathrm{e}^{-}$ densities produced by the central pulsar that exceed the standard interstellar medium energy density. Thus, nearly all systems that are classified as $\mathrm{TeV}$ halos by the latter definition are also $\mathrm{TeV}$ halos in the former definition, but the converse is not true.

We argue for the former definition, noting that it is more observationally focused. The diffusive/advective propagation of cosmic-rays can be directly measured via the $\mathrm{r}^{-1}$ vs. $\mathrm{r}^{-2} \gamma$-ray morphology. The comparison of the source energy density to the interstellar medium energy density, on the other hand, must rely on ISM models that can not be measured from $\gamma$-ray data.

Either way, to understand most of the $\mathrm{TeV}$ halo literature, it is imperative to determine which definition is being used. Notably, the brightest individual systems, as well as the majority of the total leptonic $\gamma$-ray flux, is produced by systems with ages between 20-100 kyr, which are termed $\mathrm{TeV}$ halos in the former definition but "extended PWN" in the latter. Thus, multiple statements in the literature, such as the fact that $\mathrm{TeV}$ halos produce the majority of the diffuse Milky Way $\gamma$-ray flux, depend sensitively on the definition being used.

This being said - the physics is invariant. Regardless of the definition employed in the literature - the observation that young and middle-aged pulsars efficiently convert their spindown power into relativistic $\mathrm{e}^{+} \mathrm{e}^{-}$pairs that lose a significant fraction of their energy through inverse-Compton scattering that produces $\gamma$-rays with energies up to at least $100 \mathrm{TeV}$ in diffuse regions immediately surrounding the pulsar sources remains unchanged. These observations have significant implications for our understanding of high-energy astrophysics. 


\section{Implications of TeV Halos}

For the remainder of this article, we will adopt the former definition of $\mathrm{TeV}$ halo, which indicates that the majority of $20-100 \mathrm{kyr}$ old pulsars power $\mathrm{TeV}$ halos in addition to compact PWN. We note that, if the latter definition were adopted instead, every implication remains true, under a replacement of the words "TeV halo" with "TeV halos and extended PWN".

\subsection{The Majority of Diffuse TeV $\gamma$-ray Emission is Produced by TeV Halos}

If pulsars efficiently convert a significant fraction of their total spin-down power to $\mathrm{e}^{+} \mathrm{e}^{-}$pairs, and those $\mathrm{e}^{+} \mathrm{e}^{-}$pairs subsequently lose energy in regions of the interstellar medium where the magnetic field energy density and interstellar radiation energy density are approximately equal (as is true throughout diffuse regions of the Milky Way) - then the conclusion that $\mathrm{TeV}$ halos dominate the diffuse $\gamma$-ray emission from the Milky Way is inescapable.

This diffuse $\gamma$-ray emission stems from a combination of two sources: (1) the TeV halos of sources that are too dim to be individually detected by current instruments, (2) the extremely diffuse emission from $\mathrm{e}^{+} \mathrm{e}^{-}$pairs that escape from the $\mathrm{TeV}$ halo but continue to produce $\gamma$-rays through the interstellar medium via inverse-Compton scattering. The former contribution is expected to be dominant, though the exact ratio depends on the effectiveness of $\mathrm{e}^{+} \mathrm{e}^{-}$cooling within $\mathrm{TeV}$ halos, a parameter that is degenerate with the value of the $\mathrm{e}^{+} \mathrm{e}^{-}$conversion efficiency of pulsar magnetospheres.

In Ref. [10] we incorporate models for the birth-rate, distribution and spin-down power of the Milky Way pulsar population. Assuming that the average pulsar has a $\sim 10 \% \mathrm{e}^{+} \mathrm{e}^{-}$injection efficiency (similar to best-fit models for Geminga), we find that the population of individually unresolved $\mathrm{TeV}$ halos produces a diffuse $\mathrm{TeV} \gamma$-ray signal that outshines supernova remnants (which have much softer spectra). Intriguingly the total flux of these sources are consistently with the diffuse $\mathrm{TeV}$ excess, previously discovered by the Milagro collaboration. Further observations by HAWC and LHAASO will be capable of further resolving this underlying population and validating $\mathrm{TeV}$ halo models for the diffuse TeV sky.

Intriguingly, these same models also predict a significant diffuse synchrotron emission

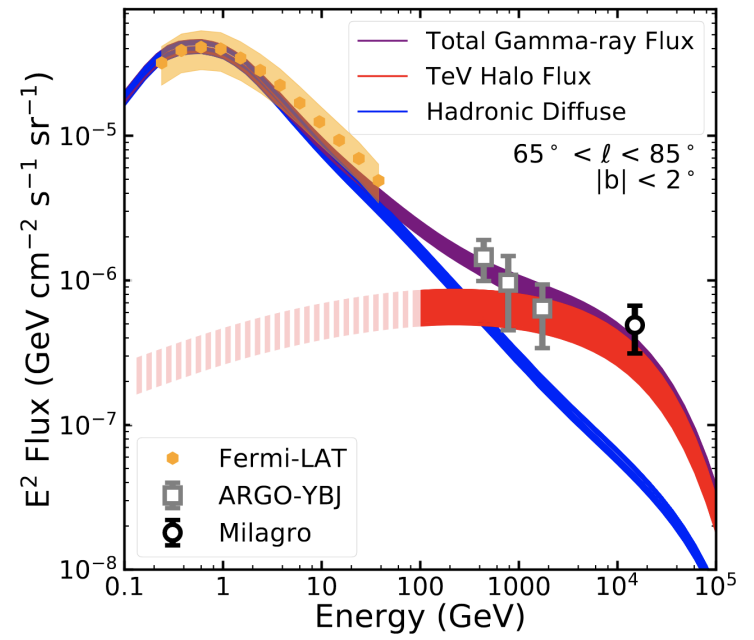

Figure 1: The relative contribution of diffuse hadronic $\gamma$-rays (from supernova remnants) and diffuse leptonic $\gamma$-rays (from $\mathrm{TeV}$ halos) to the diffuse $\gamma$-ray emission across the solar plane. While the $\mathrm{TeV}$ halo contribution is subdominant at $\mathrm{GeV}$ energies, its hard spectrum makes it dominate the $\mathrm{TeV} \gamma$-ray flux. From Ref. [10]. - with hard spectra that are consistent with the hard spectrum of $\mathrm{TeV}$ halos. At present, this synchrotron signal currently falls below the sensitivity of wide-field instruments capable of detecting a diffuse signal. However, future instruments may be capable of using synchrotron observations to validate $\mathrm{TeV}$ halo measurements and better constrain the relative magnetic field and interstellar radiation field energy densities within halos. 


\subsection{The Majority of TeV $\gamma$-ray Sources are $\mathrm{TeV}$ halos}

At present, only a handful of $\mathrm{TeV}$ halos have been definitively detected by the HAWC, H.E.S.S. and LHAASO collaborations. An additional, more significant population of "TeV PWN", originally identified by the H.E.S.S. collaboration, would be described as $\mathrm{TeV}$ halos under our adopted definition. In sum, these sources correspond to approximately $1 / 3$ of the $\mathrm{TeV}$ sources detected by current instrumentation ${ }^{1}$. However, they are biased towards the most distant and powerful sources, primarily because H.E.S.S. observations are unable to detect any source with an angular extent exceeding $\sim 0.6^{\circ}$, making the population of nearby, middle-aged pulsars observable only with the less sensitive HAWC survey. Future HAWC and LHAASO observations will significant improve the completness of these searches, as shown in Fig. 2.

However, these sources are only the tip of the iceberg. At present, a source can only be identified as a $\mathrm{TeV}$ halo if the associated pulsar is detected and identified using radio or $\gamma$-ray observations. However, approximately $70 \%$ of middle-aged pulsars do not have radio beams that are oriented towards Earth, making their detection much harder, and rendering the sources unidentified. Notably, this syncs well with curent H.E.S.S catalog observations, which find that slightly more than half of all H.E.S.S. soruces are unassociated with any known astrophysical object.

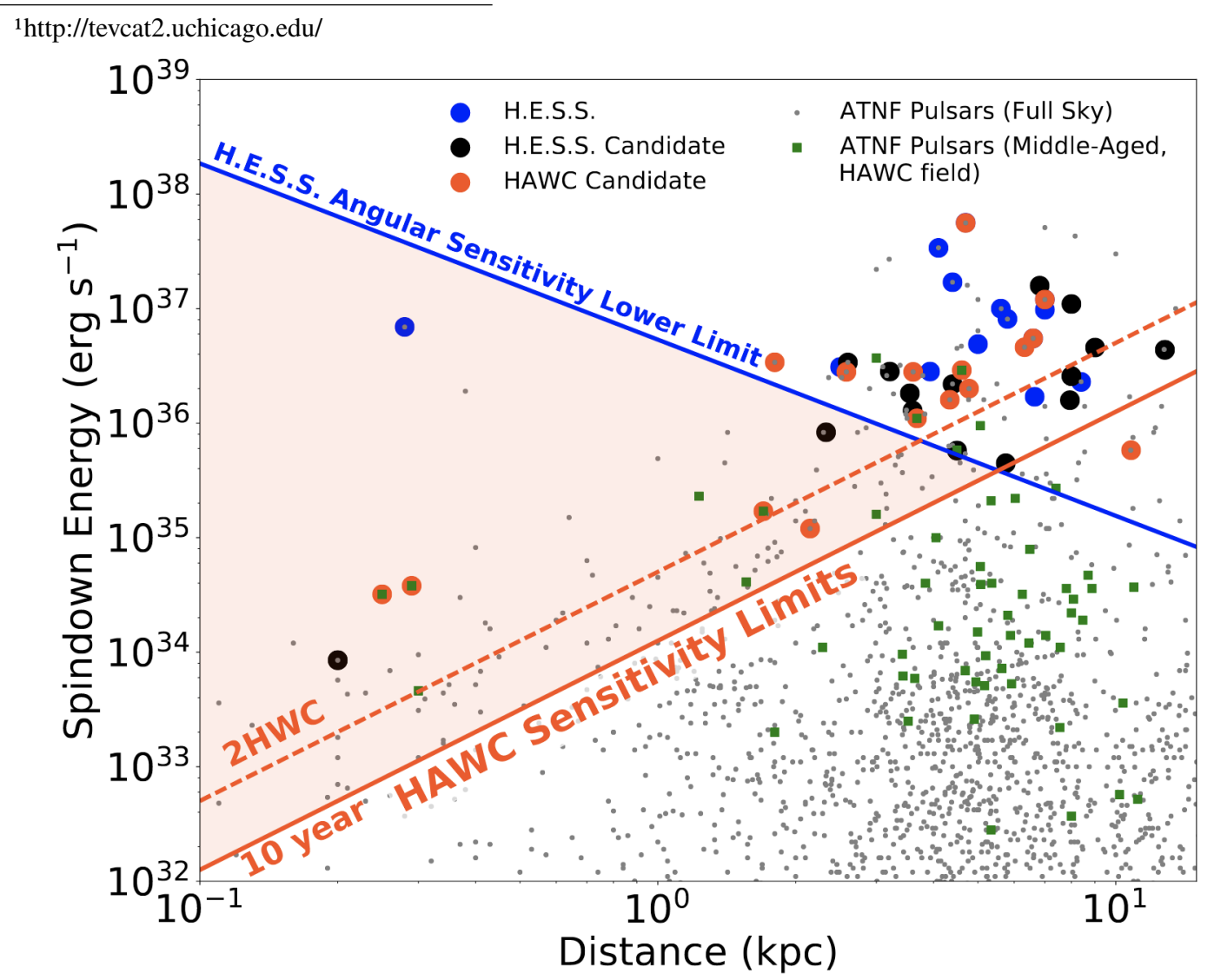

Figure 2: The parameter space of $\mathrm{TeV}$ halo observations in terms of the spindown power and source distance (which determines the angular size of the source). HAWC observations (and upcoming LHAASO observations) are particularly sensitive to the population of nearby, middle-aged pulsars, which would escape the detection of atmospheric Cherenkov telescopes due to their large angular size. Details in Ref. [6]. 


\subsection{Pulsars Power the Rising Positron Excess}

Our first conclusion (that $\mathrm{TeV}$ halos dominate the diffuse $\mathrm{TeV}$ flux) depends only on the observed fact that $\mathrm{TeV}$ halos efficiently convert a large fraction of their spindown power to $\mathrm{e}^{+} \mathrm{e}^{-}$pairs. Our second conclusion (that most sources are $\mathrm{TeV}$ halos) additionally depends on the observation that these $\mathrm{TeV} \mathrm{e} \mathrm{e}^{+} \mathrm{e}^{-}$pairs lose a significant fraction of their energy before escaping the $\mathrm{TeV}$ halo. Our final conclusion concerns only the $\mathrm{GeV} \mathrm{e} \mathrm{e}^{+}$pairs, and depends on models indicating that lower energy positrons travel farther before losing their energy, and thus escape the $\mathrm{TeV}$ halo entirely.

This conclusion is well founded based on two observations. The first, by Ref. [11] shows that the $\mathrm{GeV}$ emission from $\mathrm{TeV}$ halos extended to significantly larger distances than the observed $\mathrm{TeV}$ emission. This is theoretically expected, because the cooling timescale for electrons falls rapidly as $\mathrm{E}^{-1}$, while the efficiency of electron diffusion increases only as $\sim \mathrm{E}^{0.33}$.

The second observable fact is the decadeold mystery of the unexpected rise in the positron fraction detected by PAMELA, FermiLAT and AMS-02. Even before the observation of $\mathrm{TeV}$ halos, pulsars were consider to be a leading explanation for the positron excess. At this time, the largest uncertainty was the fraction of the pulsar spindown power which was converted into $\mathrm{e}^{+} \mathrm{e}^{-}$pairs. Models indicates that this efficiency must be at least $1 \%$ (and potentially as much as $10 \%$ ) to explain the properties of the excess. Excitingly, TeV halo observations have validated this efficiency, solving the primary concern regarding pulsar explanations of the excess $[5,12]$

Several authors, however, including the HAWC collaboration - have considered a new

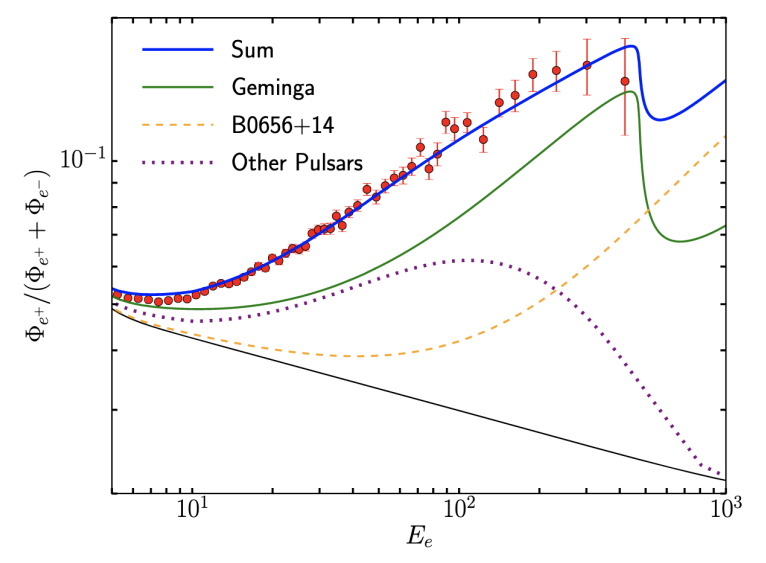

Figure 3: The contribution of Geminga, Monogem, and the sum of more distant pulsars to the observed positron fraction at Earth. Models with $\mathrm{e}^{+} \mathrm{e}^{-}$efficiencies near $10 \%$, and spectra consistent with $\mathrm{TeV}$ halo $\gamma$-ray observations can precisely fit the main properties of the positron excess. From Ref. [5]. potential complexity. What if $\mathrm{GeV}$ positrons (like $\mathrm{TeV} \mathrm{e} \mathrm{e}^{+} \mathrm{e}^{-}$pairs) do not efficiently travel from the $\mathrm{TeV}$ halo all the way to Earth. Such a scenario is possible, if the region of low-diffusion surrounding the $\mathrm{TeV}$ halo extends significantly farther than the $20 \mathrm{pc}$ region over which the $\mathrm{TeV} \gamma$-ray signal is observed [2]. However, such a scenario is highly unlikely for several reasons: (1) energetically, the $\mathrm{TeV}$ halo is incapable of changing the turbulence in the galactic magnetic field on $\sim 100 \mathrm{pc}$ scales, in fact even $20 \mathrm{pc}$ halos are challenging from an energetics perspective, (2) the average diffusion coefficient of the galaxy would be significant affected if TeV halos extended on $\sim 100 \mathrm{pc}$ scales, producing tension with standard observations of primary-to-secondary ratios by AMS-02 [13], (3) the local diffusion constant near Earth is high, as evidenced by the diffuse $\mathrm{TeV}$ electron flux which is observed at Earth today [13, 14]. For these three reasons, the extent of $\mathrm{TeV}$ halos is unlikely to exceed $20 \mathrm{pc}$, making it highly likely that $\mathrm{GeV}$ positrons leach out of the halos and propagate to Earth, where they produce the rise in the observed positron fraction. 


\section{Conclusions}

In this short article, we have focused on the status of $\mathrm{TeV}$ halo observations, and determined their immediate consequences for our understanding of $\mathrm{TeV}$ astrophysics. This, however, is only the first step. Detailed models of TeV halo formation and evolution are necessary in order to understand the complex interplay between the supernova remnant, PWN, and halo.

The low diffusion constant observed within $\mathrm{TeV}$ halos is of particular interest, as its origin is unknown. One promising mechanism is the self-generation of turbulence through cosmic-ray propagation. At present, this phenomenon has only been discussed within compact sources such as supernova remnants. $\mathrm{TeV}$ halos offer the potential to allow us to better understand the interaction between these compact sources and the surrounding interstellar medium [15-17].

Additionally, $\mathrm{TeV}$ halo observations have significant implications for fundamental studies in neutrino physics and dark matter indirect detection. The application to neutrino physics is straightforward. If the primary source of $\mathrm{TeV} \gamma$-rays from Milky Way-like galaxies is leptonic in nature, the expected neutrino flux from the Milky Way and similar star-forming galaxies is significantly lessened. Such a scenario tilts the balance towards radio galaxy and AGN explanations for the IceCube extragalactic neutrino background.

The application of $\mathrm{TeV}$ halo studies to dark matter is less straightforward - but similarly important. We have reached a new frontier in dark matter studies, where the precision of current cosmic-ray and $\gamma$-ray experiments are outpacing our ability to model cosmic-ray acceleration and propagation throughout the Galaxy. New phenomena, like inhomogeneous diffusion, likely affect our measurements of the local cosmic-ray spectrum at the $1 \%$ level, which is well within the sensitivity of current dark matter experiments. $\mathrm{TeV}$ halos potentially provide us with a new precision tool to study the local effects of cosmic-ray diffusion in regions of space that were previously considered to be a portion of the undisturbed ISM. New models, observations, and theories regarding $\mathrm{TeV}$ halo formation and evolution may provide the key to improve our probes of dark matter phenomenology.

\section{References}

[1] A. U. Abeysekara et al., Astrophys. J. 843, 40 (2017), arXiv:1702.02992 [astro-ph.HE] .

[2] A. U. Abeysekara et al. (HAWC), Science 358, 911 (2017), arXiv:1711.06223 [astro-ph.HE] .

[3] H. Yuksel, M. D. Kistler, and T. Stanev, Phys. Rev. Lett. 103, 051101 (2009), arXiv:0810.2784 [astro-ph] .

[4] H. Abdalla et al. (HESS), Astron. Astrophys. 612, A2 (2018), arXiv:1702.08280 [astro-ph.HE]

[5] D. Hooper, I. Cholis, T. Linden, and K. Fang, Phys. Rev. D 96, 103013 (2017), arXiv:1702.08436 [astro-ph.HE] .

[6] T. Linden, K. Auchettl, J. Bramante, I. Cholis, K. Fang, D. Hooper, T. Karwal, and S. W. Li, Phys. Rev. D 96, 103016 (2017), arXiv:1703.09704 [astro-ph.HE] . 
[7] T. Sudoh, T. Linden, and J. F. Beacom, Phys. Rev. D 100, 043016 (2019), arXiv:1902.08203 [astro-ph.HE] .

[8] F. A. Aharonian, Nucl. Phys. B Proc. Suppl. 39, 193 (1995).

[9] G. Giacinti, A. M. W. Mitchell, R. López-Coto, V. Joshi, R. D. Parsons, and J. A. Hinton, Astron. Astrophys. 636, A113 (2020), arXiv:1907.12121 [astro-ph.HE] .

[10] T. Linden and B. J. Buckman, Phys. Rev. Lett. 120, 121101 (2018), arXiv:1707.01905 [astroph.HE] .

[11] M. Di Mauro, S. Manconi, and F. Donato, Phys. Rev. D 100, 123015 (2019), arXiv:1903.05647 [astro-ph.HE] .

[12] S. Profumo, J. Reynoso-Cordova, N. Kaaz, and M. Silverman, Phys. Rev. D 97, 123008 (2018), arXiv:1803.09731 [astro-ph.HE] .

[13] D. Hooper and T. Linden, Phys. Rev. D 98, 083009 (2018), arXiv:1711.07482 [astro-ph.HE] .

[14] H. Abdalla et al. (H.E.S.S.) (2017) arXiv:1709.06442 [astro-ph.HE] .

[15] C. Evoli, T. Linden, and G. Morlino, Phys. Rev. D 98, 063017 (2018), arXiv:1807.09263 [astro-ph.HE] .

[16] K. Fang, X.-J. Bi, and P.-F. Yin, Astrophys. J. 884, 124 (2019), arXiv:1906.08542 [astroph.HE] .

[17] G. Johannesson, T. A. Porter, and I. V. Moskalenko, Astrophys. J. 879, 91 (2019), arXiv:1903.05509 [astro-ph.HE] . 